\title{
Heat-fixation Method Used in an Atomic Force Microscopy Study of Cell Morphology
}

\author{
Zhe-Xue Lu,* Zhi-Ling Zhang, ${ }^{*}$ Wan-Liang SHI,** Dai-Wen PAng, ${ }^{* \dagger}$ Zhi-Xiong XIE, ** \\ and Ping Sheng** \\ * College of Chemistry and Molecular Sciences and State Key Laboratory of Virology, \\ Wuhan University, Wuhan 430072, P. R. China \\ ** College of Life Sciences, Wuhan University, Wuhan 430072, P. R. China
}

\begin{abstract}
In order to overcome the difficulties with existing methods for sample immobilization in imaging Halobacterium salinarum (H. salinarum) living in a highly salty medium by atomic force microscopy (AFM), a heat-fixation method was, for the first time, used to overcome existing problems in preparing samples for AFM. The effect on the cell morphology of the heat-fixation method was studied by MAC mode AFM, and was compared with the drop-and-dry and the polylysine-adhesion methods. It was found that the heat-fixation method can be successfully used for preparing Gram-negative and Gram-positive bacteria samples for AFM studies. Using this method, high-resolution AFM images of $H$. salinarum were obtained. Round protrusions on the cell surface and horn-like protrusions only at one pole of $H$. salinarum were observed.
\end{abstract}

(Received April 23, 2007; Accepted July 25, 2007; Published February 10, 2008)

\section{Introduction}

Extremely halophilic archaea are a diverse group of prokaryotes that inhabit highly saline environments. H. salinarum, a species of archaebacteria, is a photosynthetic bacteria that can survive in $4 \mathrm{M} \mathrm{NaCl}$ solutions. When the concentration of $\mathrm{Na}^{+}$is low, the cell wall will break apart and the cell will be lysed. ${ }^{1}$ To date, the reason why $H$. salinarum requires a high concentration of sodium ions for growth has not been fully explained. Archaebacteria have a different morphology than other prokaryotes, such as E. coli. ${ }^{2,3}$ As is known, the functions of the cells are related to the structure of the cell membrane. Thus, investigations of the structure of the $H$. salinarum surface are quite significant. Atomic force microscopy (AFM) is a nondestructive imaging technique with nanometer resolution. Since its introduction in 1986, AFM has been used more extensively in the field of life sciences. ${ }^{4-6}$ AFM images can reveal the surface ultrastructure of living microbial cells with unprecedented resolution..$^{7-9}$

AFM can be operated in several operational modes, including contact, tapping and MAC modes. ${ }^{10}$ In the MAC mode, an alternating magnetic field, generated by a solenoid placed under the sample, is used to directly drive a magnetically coated cantilever to enable a stable operation. The advantages of the MAC mode are high resolution and easy operation in fluids for in situ studies, and little harm to soft biological samples due to intermittent contact.

For AFM imaging, samples must be immobilized on a substrate. A variety of methods have been used to immobilize cells for AFM imaging, including trapping living cells in filtrate films, placing cells in agar, or immobilizing cells onto a surface such as mica or glass. ${ }^{4}$ In microbiology, the heat-fixation method is widely used for immobilizing bacterial cells onto

$\dagger$ To whom correspondence should be addressed. glass slides for observing cell morphology by optical microscopy. For low-resolution observations with an optical microscope, it seems that this method does not affect the cell morphology. However, the effect of this method on the ultrastructure of the cell morphology has not been examined.

Herein, the effect of the heat-fixation method on cell morphology studied by MAC mode atomic force microscopy is reported. Escherichia coli (E. coli) and Bacillus subtilis (B. subtilis) were investigated, respectively, as representatives of Gram-negative bacteria and Gram-positive bacteria. The heatfixation method was then employed to prepare $H$. salinarum samples for AFM study. Details of the ultrastructural morphology of H. salinarum surface were obtained by MAC mode AFM.

\section{Experimental}

\section{Culture of bacteria}

E. coli JM109 and B. subtilis DB104 were grown in LuriaBertani (LB). H. salinarum $\mathrm{R} 1$ was grown in a medium containing $0.25 \%$ lactalbumin hydrolysate, $0.2 \%$ yeast extract, $25 \% \mathrm{NaCl}$ and $3 \%$ magnesium chloride heptahydrate at $\mathrm{pH} 7.0$. The three species of bacteria were cultured at $37^{\circ} \mathrm{C}$ with shaking and harvested in the late exponential phase of growth. Bacteria were centrifuged at $5000 \mathrm{rpm}$ for $4 \mathrm{~min}$, and the wet pellets were resuspended in normal saline for E. coli and B. subtilis and $4 \mathrm{M} \mathrm{NaCl}$ solution for $H$. salinarum, respectively, and were recentrifuged at $5000 \mathrm{rpm}$ for $4 \mathrm{~min}$ to remove the growth medium. The final pellets were again resuspended in normal saline and $4 \mathrm{M} \mathrm{NaCl}$ solution, respectively.

Immobilization of bacteria on glass slides by the heat-fixation method

Glass slides were pretreated with concentrated $\mathrm{H}_{2} \mathrm{SO}_{4}$, washed 3 times with ultrapure water, and then dried by $\mathrm{N}_{2}$. Bacterial 

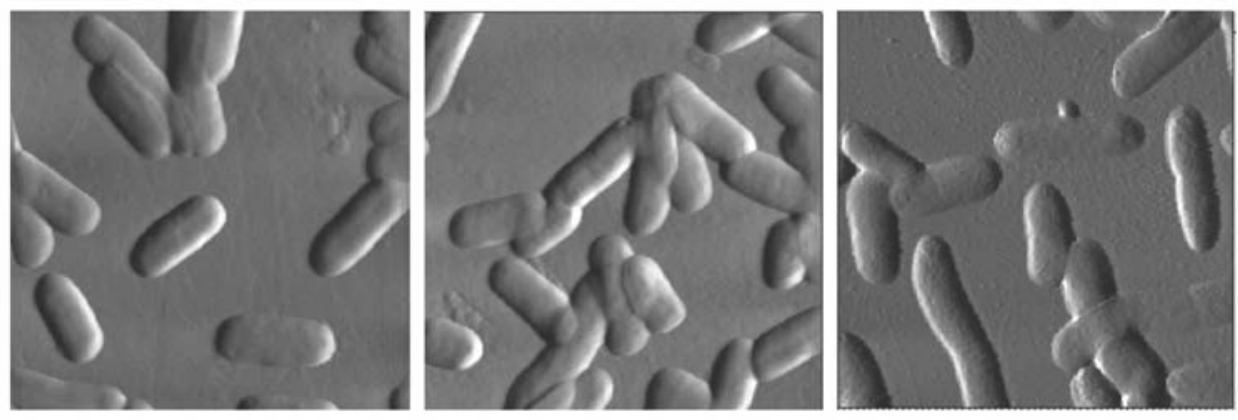

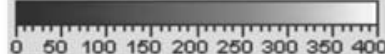

A

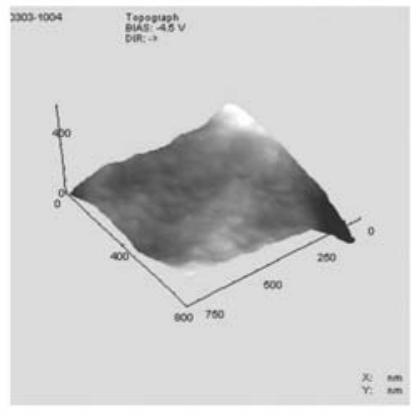

D

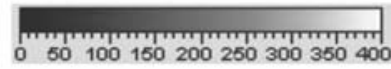

B

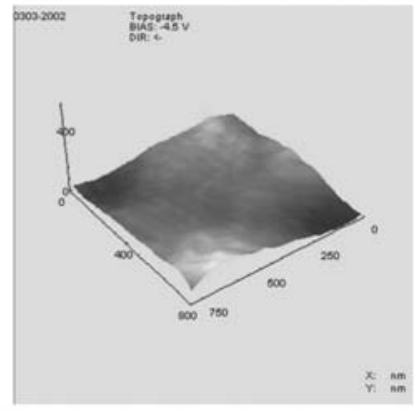

E

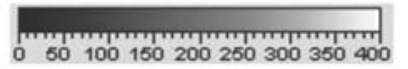

C

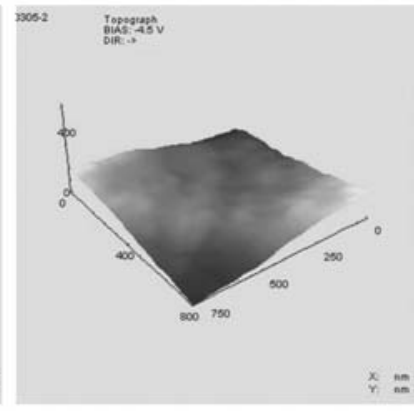

F

Fig. 1 Topography mode (A) - (F) AFM images of E. coli treated by the drop-and-dry method (A), heat-fixation method (B) and the poly-L-lysine-adhesion method (C). (D) - (F): 3D images acquired by zooming in on a desired cell in (A) - (C), respectively. Image sizes: (A) - (C) $12 \times 12 \mu \mathrm{m}^{2}$, (D) (F) $800 \times 800 \mathrm{~nm}^{2}$.

solutions were dropped onto glass slides, dried by moving backwards and forwards over a Bunsen flame two or three times rapidly, subsequently rinsed with ultrapure water, and then dried by $\mathrm{N}_{2}$.

\section{Atomic force microscopy}

Images were acquired using a PicoScan system with the MAC mode (Molecular Imaging, USA). $\mathrm{Si}_{3} \mathrm{~N}_{4}$ cantilevers covered with a magnetic film (MAClever TypeII, Molecular Imaging) were employed. The scanner used was an AFM "M" piezo one with a maximum scan range of $30 \times 30 \mu \mathrm{m}^{2}$ and a maximum $z$-range of $4 \mu \mathrm{m}$. All images were obtained in the topography mode. Then, $10 \mu \mathrm{l}$ of a bacterial suspension was dropped onto $2 \times 2 \mathrm{~cm}^{2}$ poly-L-lysine coated glass slides ${ }^{11}$ or $2 \times 2 \mathrm{~cm}^{2}$ glass slides, and the later were heat-fixed or air-dried. AFM imaging was conducted in solution.

\section{Results and Discussion}

Among the existing immobilization methods for AFM imaging, the most commonly used method is the poly-L-lysine-adhesion method, by which samples are immobilized through electrostatic interaction, and keep their original morphology and state. Another is the drop-and-dry method, in which samples are immobilized by evaporating the solution to form a film..$^{11,12}$ However, because air-drying needs a relatively long period of time, bacterial cells are difficult to maintain their original living state. Nevertheless, the heat-fixation method can help retain bacterial morphology by coagulating the cytoplasm and enhance the adhesion of cells to glass slides via coagulation of surface proteins of bacteria onto the glass slides. The feasibility of using this method for preparing bacterial cell samples for AFM studies was investigated by comparing it with the drop-and-dry method and the poly-L-lysine-adhesion method.

The influence of heat-fixation on the morphology of E. coli (Gram-negative bacteria)

Bacteria can be divided into two major groups, namely Gramnegative and Gram-positive. The Gram-negative cell wall is made of a complicated multilayered structure. Five to twenty percent of the cell wall is composed of peptidoglycan, and the remainder consists of lipopolysaccharides, which reside in the outer-membrane layer outside the peptidoglycan layer. ${ }^{1}$ E. coli was chosen as a representative of Gram-negative bacteria for the present study.

AFM images of $E$. coli samples prepared by the drop-and-dry method are shown in Fig. 1A. Figure 1A is a topography image, giving quantitative topographic information. Individual E. coli bacterium could be clearly distinguished. As shown in Fig. 1A, the length of $E$. coli was about $2.5 \mu \mathrm{m}$, and the width and height were 1.2 and $0.25 \mu \mathrm{m}$, respectively (ten cells were measured). Figures $1 \mathrm{~B}$ and $1 \mathrm{C}$ show AFM images of E. coli samples immobilized by the heat-fixation method and the polylysine-adhesion method. It is obvious that the length, width and height hardly changed. Detailed structure of the cell wall was acquired by zooming in on regions of the cell envelope (Figs. 1D - 1F). The surface morphology and roughness were 

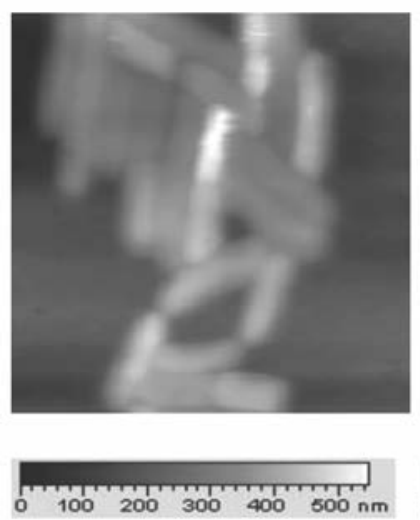

A

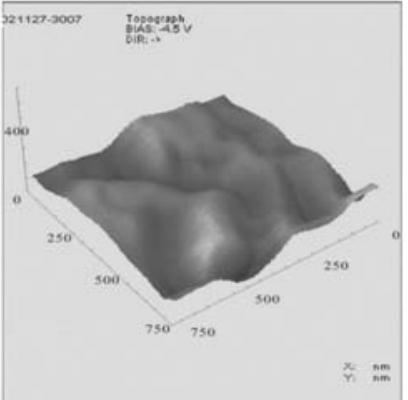

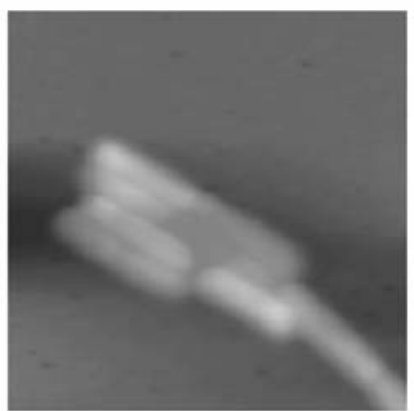

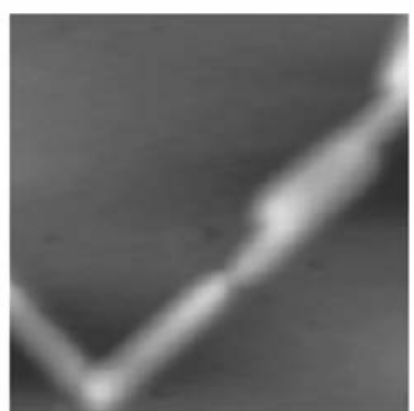

$100200300400500 \mathrm{~nm}$

C

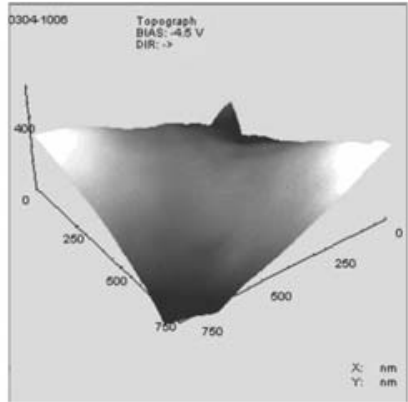

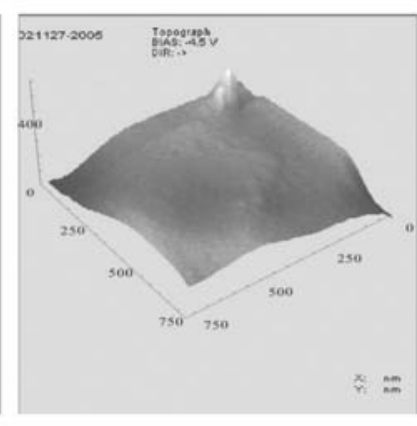

F

Fig. 2 Topography mode (A) - (F) AFM images of B. subtilis treated by the drop-and-dry method (A), heat-fixation method (B) and the polylysine-adhesion method (C). (D) - (F): 3D images acquired by zooming in on a desired cell in $(A)-(C)$, respectively. Image sizes: $(A)-(C) 10 \times 10$ $\mu \mathrm{m}^{2}$, (D) $-(\mathrm{F}) 800 \times 800 \mathrm{~nm}^{2}$.

nearly the same with the three different immobilization methods.

The effect of heat-fixation on the morphology of B. subtilis (Gram-positive bacteria)

The cell sizes of $B$. subtilis samples prepared by the three different immobilization methods suggest that the average sizes of $B$. subtilis with the heat-fixation (width, $820 \pm 20 \mathrm{~nm}$; height, $300 \pm 10 \mathrm{~nm}$ ) were nearly the same as those obtained with the drop-and-dry immobilization method (width, $800 \pm 10 \mathrm{~nm}$; height, $320 \pm 20 \mathrm{~nm}$ ) and the poly-L-lysine-adhesion method (width, $800 \pm 30 \mathrm{~nm}$; height, $330 \pm 30 \mathrm{~nm}$ ).

However, when comparing the surface morphologies of $B$. subtilis samples prepared by the three different immobilization methods in highly-resolved 3D images, it was found that ridgelike structures and larger surface roughness were observed on the surface of $B$. subtilis with the drop-and-dry method, while the surface was smooth with the heat-fixation method and the poly-L-lysine-adhesion method (see Fig. 2D with Figs. 2E and 2F). The cell-wall structure of Gram-positive bacteria is quite different from the cell wall of Gram-negative bacteria. Unlike Gram-negative bacteria, Gram-positive bacteria possess only a single thick peptidoglycan layer containing covalently linked anionic polymers, and thus lack a true periplasm. The structure of the relatively thick, highly charged wall changes with changing the local environment of the Gram-positive cell membrane. ${ }^{13}$ In view of physiology, B. subtilis would form a dry state, in which the cells could not move, and their metabolic processes would be dramatically slowed down, because of water evaporation during drop-and-dry immobilization; then, the cell wall structure might accordingly change. This might be partially responsible for the protrusions and the ridge-like structures. On the contrary, the heat-fixation method could keep the morphology of the cell surface close to the original structure of living cells because of rapid immobilization.

\section{Application of the heat-fixation method in $H$. salinarum} (archaebacteria) immobilization

Extremely halophilic archaea are also classified as Grampositive or Gram-negative. Thus the heat-fixation method was used to prepare $H$. salinarum samples for AFM studies.

Imaging $H$. salinarum in solution by immobilizing on poly-Llysine coated glass slides ${ }^{11}$ was tried, but failed. The reason for this might be that the concentration of the salts in the bacterial sample was high, which could lower the electrostatic interaction between the negatively-charged bacteria and positively-charged poly-L-lysine, so the bacteria could not adhere strongly to the substrate. The drop-and-dry method, as described by Butt et $a l .{ }^{12}$ was also tried, but there were too many salt crystals on the glass slide, which affected the AFM imaging. Therefore, the heat-fixation method was tried to immobilize $H$. salinarum on glass slides for AFM imaging.

A series of AFM images of $H$. salinarum samples prepared by the heat-fixation method are shown in Figs. 3A - 3C. An individual bacterium was distinguishable with the typical length and height of a bacterial cell of about $0.9 \mu \mathrm{m}$ and $120 \mathrm{~nm}$, respectively. There were some round protrusions on the membrane surface (shown with an arrow in Fig. 3D) and several horn-like protrusions only at one pole of the cell (see Figs. 3A 3C). Repeated imaging experiments gave consistent results. 

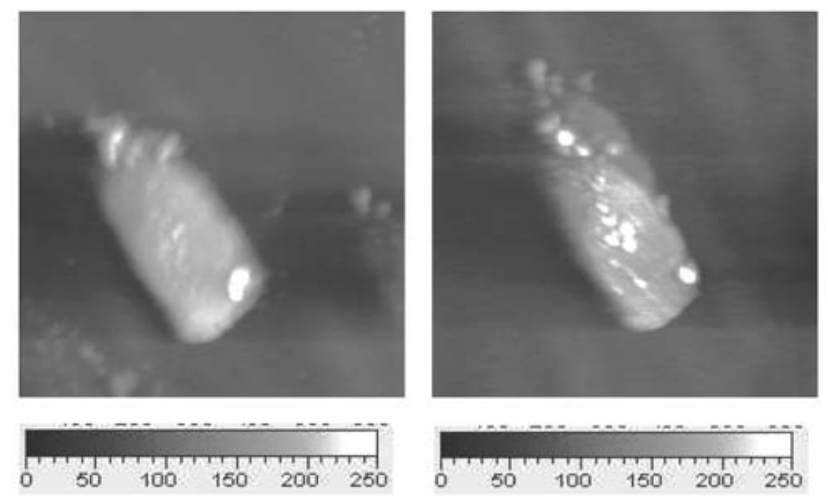

A

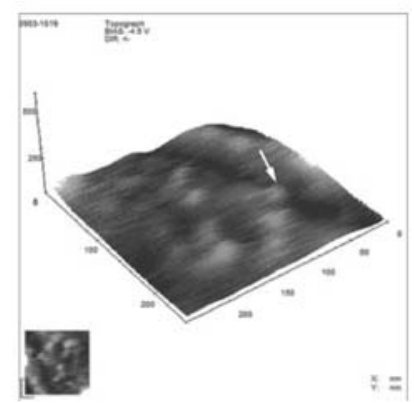

D
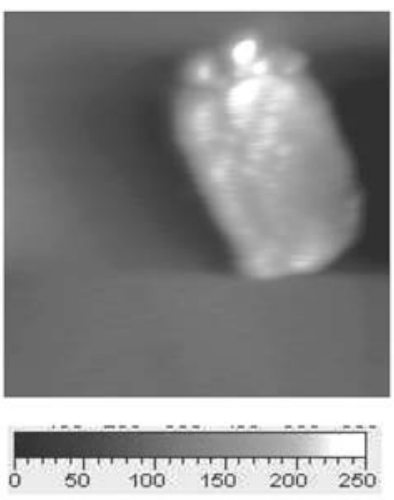

C

Fig. 3 Series of topography mode (A) - (C) AFM images of $H$. salinarum treated by the heatfixation method. (D) 3D image acquired by zooming in on the cell in (C). Image sizes: (A) - (C) 1.2 $\times 1.2 \mu \mathrm{m}^{2}$, (D) $250 \times 250 \mathrm{~nm}^{2}$.

As is known, there are a great number of acidic amino acids in Halobacterium cell walls; the round protrusions could represent patches of hundreds to thousands of acidic amino acids. The horn-like protrusions demonstrated that the bacterial cell has asymmetry, as observed by Butt et al..$^{12}$

\section{Conclusions}

Based on our obtained results, the heat-fixation method is feasible for the immobilization of cells on glass slides for AFM imaging. This method was used to immobilize H. salinarum and new detailed structural information about the morphology of $H$. salinarum surface was acquired by MAC mode AFM. Round protrusions on the cell surface and horn-like protrusions only at one pole of $H$. salinarum were observed. The present research has provided a new method for cell immobilization in AFM studies.

\section{Acknowledgements}

This work was supported by the National Science Fund for Distinguished Young Scholars (Grant No. 20025311) and National Natural Science Foundation of China (Grant Nos. 20299034, 20207005, 20621502).

\section{References}

1. T. D. Brock and M. T. Madigan, "Biology of
Microorganisms", 1991, Prentice Hall.

2. S. Baron, "Medical Microbiology", 1986, Addison-Wesley, Amsterdam.

3. W. A. Volk and M. F. Wheeler, "Basic Microbiology", 1988, Harper \& Row, New York.

4. T. A. Camesano, M. J. Natan, and B. E. Logan, Langmuir, 2000, 16, 4563.

5. T. C. Pesacreta, L. C. Carlson, and B. A. Triplett, Planta, 1997, 202, 435.

6. J. C. Thimm, D. J. Burritt, W. A. Ducker, and L. D. Melton, Planta, 2000, 212, 25.

7. Y. F. Dufrene, C. J. P. Boonaert, P. A. Berin, and M. Asther, J. Bacteriol., 1999, 181, 5350.

8. N. A. Amro, L. P. Kotra, K. Wadu-Mesthrige, A. Bulychev, S. Mobashery, and G. Liu, Langmuir, 2000, 16, 2789.

9. Z. X. Lu, L. Zhou, Z. L. Zhang, W. L. Shi, Z. X. Xie, H. Y. Xie, D. W. Pang, and P. Shen, Langmuir, 2003, 19, 8765.

10. W. Han, S. M. Linday, and T. W. Jing, Appl. Phys. Lett., 1996, 69, 4111.

11. A. V. Bolshakova, O. I. Kiselyova, A. S. Filonov, O. Yu. Frolova, Y. L. Lyubchenko, and I. V. Yaminsky, Ultramicroscopy, 2001, 86, 121.

12. H.-J. Butt, E. K. Wolff, S. A. C. Gould, B. D. Northern, C. M. Peterson, and P. K. Hansma, J. Struct. Biol., 1990, 105, 54.

13. A. L. Sonenshein, J. A. Hoch, and R. M. Losick (ed.), "Bacillus subtilis and Other Gram-positive Bacteria: Biochemistry, Physiology and Molecular Genetics", 1993, ASM Press, Washington, D.C. 